\title{
TREATMENT OF LUMBAR INTERVERTEBRAL DISC PROLAPSE
}

\author{
By Valentine Logue, M.B., B.S., M.R.C.P., F.R.C.S. \\ Neurological Surgeon to St. George's Hospital and Royal National Orthopaedic Hospital.
}

It is now 18 years since the prolapse of a lumbar intervertebral disc came to be recognized as the common pathological cause of sciatica, and although through the intervening years the clinical features of the condition have become well known and the diagnosis a commonplace, the treatment has tended to remain a controversial subject. Recently, however, several detailed follow-up studies of large numbers of patients have been published which illustrate the results of both surgical and conservative treatment, so that it is now possible to draw accurate conclusions regarding the long-term merits of any particular line of therapy and to lay down general rules for the management of the individual case. These rules can, of course, serve only as rough guides, for the treatment of lumbar intervertebral disc prolapse is almost entirely the treatment of pain, and the emotional reaction of the individual patient to the pain he is experiencing will have a considerable influence on the management of the condition, as well as social and economic factors such as the length of time a patient can remain off work and the type of work to which he must return.

Another point which must be borne in mind is that a complete relief of symptoms either by conservative or operative methods cannot be offered to the patient, because the basic pathological condition is essentially that of degeneration and disorganization of an intervertebral joint, of which the sciatica is merely a chance complication. Surgical treatment in particular tends further to disrupt the joint and although remarkable efforts to heal, and compensate for it, may be made by nature, restoration to normal is not possible and some symptoms not infrequently remain.

In fact it is a matter for some surprise that the results of treatment can be as good as they are when one considers the sequelae of disc prolapse (Fig. I); those of a narrowed disc space, sclerosis of the adjacent vertebral surfaces with spur formation and mal-alignment of the posterior intervertebral joints; to these must be added the changes which are often present in the affected nerve root conis sisting of distortion and thickening of the rootw owing to intrinsic fibrosis from tension and ischaemia, and adhesions to the damaged disc.

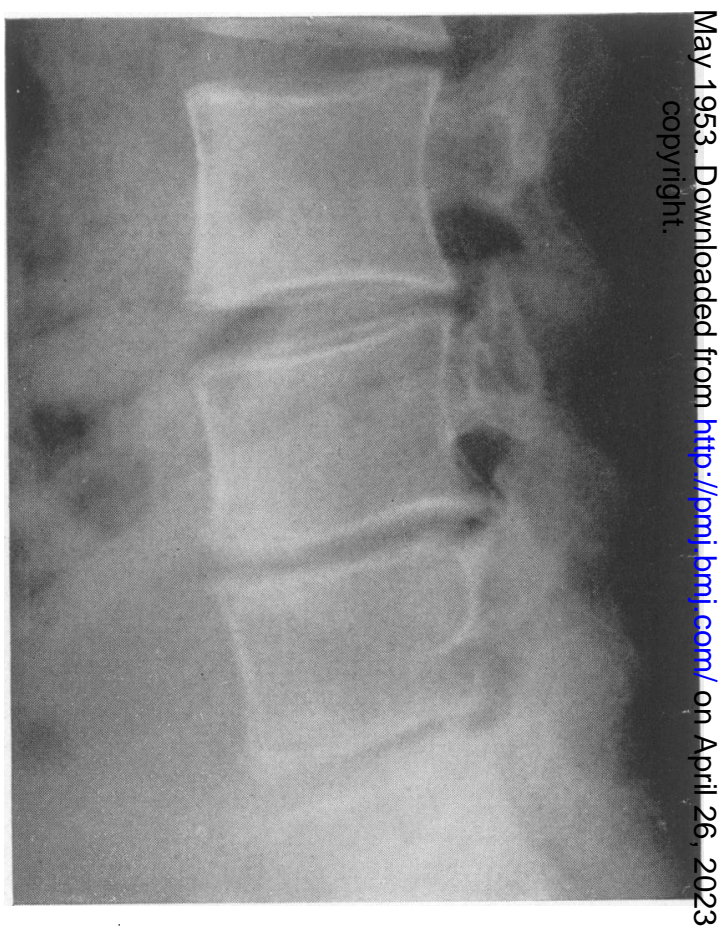

Fig. I.-X-ray of the lumbar spine of a patient wit sciatica, who had been treated conservatively, t 8 show the late results of disc degeneration. The L 4/5 disc space is narrowed and there is spur for mation from the bodies of the adjacent vertebrae. The spine shows slight angulation forwards at this level, which, combined with the approximation of the vertebrae, must disturb the alignment of th posterior intervertebral joints. 


\section{Types of Pain}

The symptoms of prolapsed lumbar intervertebral disc are predominantly those of pain, which may be felt either in the lower part of the back or in the leg or in both sites together. It is now generally accepted that sciatic pain (and anterior crural pain in higher disc lesions) is due to direct impingement of a prolapse on a nerve root and not to ' referred' pain from disorganized joints or subluxated vertebrae. Why tension on a root in its dural sheath in this situation should produce pain, whereas similar stimulation of a peripheral nerve merely produces paraesthesiae, is not accurately known, but the fact that the nerve root is exquisitely painful can easily be confirmed at operation under local analgesia.

If however, a prolapse does not happen to be so anatomically situated as to come in contact with a nerve root it can give rise solely to low back pain. The sites where this can occur are: (i) In the midline of any of the five lumbar discs (until the prolapse reaches such a size as to extend laterally to impinge on a nerve root); (ii) Laterally from the first, second or third lumbar discs, for at this high level the nerve roots arise just below the disc itself and are not involved as a rule except by the large protrusions.

In order to explain the cause of the back pain certain anatomical and physiological features of the intervertebral disc require brief mention.

\section{The Nerve Supply of the Intervertebral Disc}

The only parts of the intervertebral disc in which nerve fibres, probably subserving pain, have been demonstrated are the annulus fibrosus and the posterior longitudinal ligament (which should be regarded as part of the disc covering). These fibres arise from a recurrent branch (Fig. 2) which is given off just beyond the ganglion and returns through the intervertebral foramen to run down the spinal canal and supply the ligaments of the disc two segments below the nerve root from which it arose. At operation under local analgesia, pressure on the annulus and posterior ligament will produce the same back pain of which the patient complains, whereas the nucleus pulposus and the cartilaginous plates applied to the upper and lower surfaces of the disc are insensitive. It would seem that the back pain must have its origin in the posterior longitudinal ligament and annulus fibrosus.

The nucleus pulposus is mainly composed of water and therefore behaves as a liquid and, being virtually incompressible, performs its shockabsorber task by transmitting the considerable forces applied to it equally in all directions to the enclosing multi-layered annulus fibrosus which is the elastic structure of the disc. Degeneration or premature senescence of the nucleus pulposus and annulus fibrosus is probably an intermittently progressive affair (except in certain massive central protrusions) and only a circumscribed part of the nucleus softens at one time, tending at the instigation of trauma to be detached from the remainder of the nucleus and squeezed through a weakened area of annulus, thus coming to lie under the posterior longitudinal ligament, stretching it and its contained nerve fibres and so giving rise to low back pain.

\section{Fate of the Prolapse}

If the patient with back pain or sciatica is now treated by rest in bed or immobilization of the spine in a plaster jacket, it will frequently be found that the symptoms improve or even disappear completely. He may, if fortunate, then have no symptoms for the rest of his life, or further attacks may occur at shorter or longer intervals and the question arises as to why the pain gets better and what happens to the prolapse. Two observations have some bearing on this. Firstly, it has been shown that in patients treated by bed-rest, although the pain may clear up entirely and the spinal movements return to normal, the prolapse as revealed by myelography decreases only slightly in size. Secondly, it some-

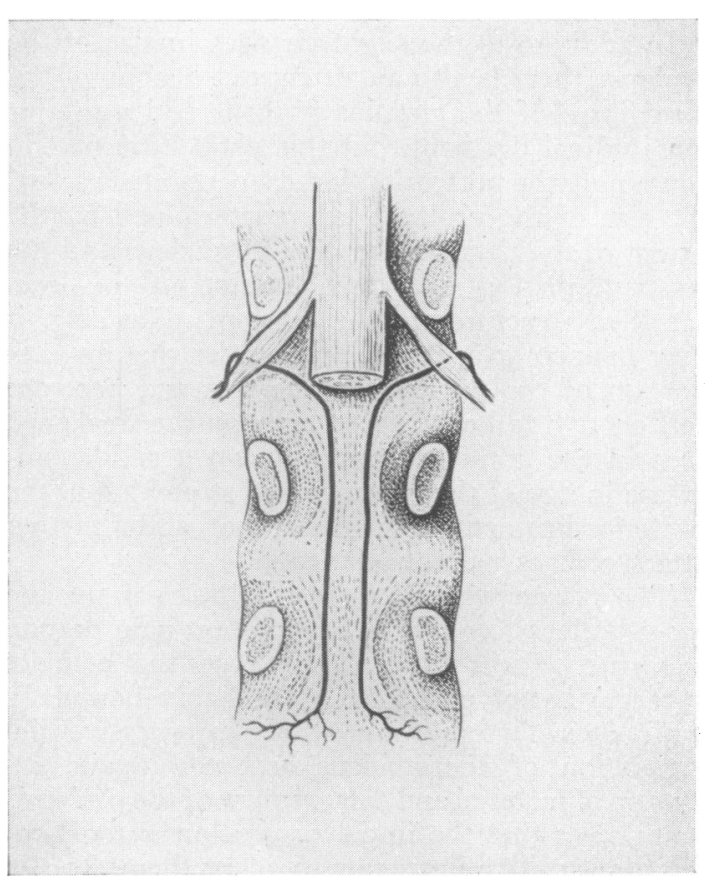

FIG. 2.-Semi-diagrammatic drawing to illustrate the course and distribution of the recurrent (sinuvertebral) nerve. 
times happens when operating on a patient with a recent disc prolapse and who has had sciatica sometime previously in the opposite leg, that if this side of the disc is exposed, a hard prolapse, often of considerable size, will be seen. The nerve root may show marked kinking around it, but despite this it apparently functions normally and certainly painlessly. It seems clear, therefore, that with a relief of symptoms the prolapse does not change greatly in size and certainly does not ' reduce itself' back within the disc space, which confirms the obvious theoretical objections to such a process taking place.

Although the main bulk of the prolapse remains there is some slight shrinkage in size and this shrinkage is due partly to reduction of tension on the disc and prolapse, as a result of recumbency and immobilization of the spine, and partly to the process of desiccation (the nuclear material is composed of 80 per cent. of water), and this seems all that is necessary to relax the tension on the posterior longitudinal ligament and so relieve the back pain. If the prolapse happens to be in contact with a nerve root producing sciatica, the slight reduction in size permits the nerve to slip off the summit of the protrusion or else gives it time to stretch and so adapt itself to the subjacent prominence. This ability of the nerve root to stretch has been confirmed experimentally in cats.

In addition to the slight changes in size of the prolapse there is also an attempt at healing of the torn fibres of the annulus fibrosus and posterior longitudinal ligament, but this plays little part in containing the protrusion for, as mentioned earlier, the size of the prolapse is predetermined by the extent of the degenerated area of nucleus and the relief of pain is due to adaptation of the local sensitive structures to the tension. However, it may happen at a variable time later that another portion of the nucleus degenerates and becomes soft enough to be squeezed through the weakened area in the annulus, so producing a sudden increase in size of the prolapse and stretching of the posterior ligament or the nerve root, and a further attack of back pain or sciatica.

The reason why all cases do not clear up and some patients are left with persistent pain despite adequate bed rest (in fact those cases that come to surgery) is not clear, but is probably bound up with such factors as a steady progressive degeneration of the nucleus and continuous extrusion of material and enlargement of the prolapse, or the fact that the initial protrusion is too large for the sensitive ligaments to adapt themselves to it. In the case of sciatic pain additional factors are concerned, i.e. the shape of the prolapse and the relationship of the nerve root to it, so that the root may be unable to slip off the summit. In other cases the nerve may be fixed to the prolapse by the formation of adhesions.

\section{Treatment}

It has been known since ancient times that be $\frac{\mathscr{P}}{\mathscr{P}}$ rest will frequently cure lumbago or sciatica, and some recent surveys of patients treated conserva흘. tively reveal that about four out of five patients with sciatica, and an even higher proportion with lumbago, will get partially or completely bettef with non-operative treatment, some permanently some to experience further attacks months or years later. In any patient therefore with $a \overrightarrow{0}$ initial attack of sciatica or lumbago, the first res course is to conservative treatment, with two exceptions: (i) The early appearance of a severe foot drop, indicating considerable damage to the motor root of one or more nerves (milder motów symptoms appear in about ro per cent. of all cases of sciatica), and (ii) A massive central extrusiobo causing a cauda equina compression. Both thesêे conditions require prompt surgery.

The important principle in conservative treat ment is immobilization of the spine and relief of weight bearing by rest in bed, which relieves tô some extent the extruding tension on the ppose lapse and so gives the ligaments or nerve root time to adapt themselves to the pressure. Immobilizag tion of the spine by a plaster jacket without rest can be used for milder cases.

Some details of bed rest require emphasizing Firstly, i: must be rigidly enforced and thi usually means admission to hospital. The patient should not get out of bed for any purpose and foo the first week he should not be permitted to sit up. The mattress should be a firm one and shoulf not sag, if necessary it can be supported bj fracture boards. Traction on the leg by means of a weight and adhesive strapping is sometimes. beneficial in some cases with very severe pain. is important that analgesic drugs be administere in adequate quantities to make the pain bearable during the early stages.

The next question is how long should bed rest be continued? Experience has shown that the majority of cases which are going to improve wit this treatment will have shown some response within three weeks, and this should be the minis mum time that the patient remains in bed. If at the expiration of this time the pain has abateg completely the patient can start getting up a once. If some symptoms still persist but it is evident that the condition is improving steadil then a further period of rest up to about four weeks is advised. If mild symptoms continue he should then get up in a plaster jacket or a support ing corset of the Goldthwaite type. In any everf 
activity should be restricted and he should avoid heavy lifting for a further three months.

On the other hand, if at the end of three weeks the symptoms have shown no signs of abating, then operative treatment will have to be considered.

This assessment of the problem after a three weeks' trial of recumbency is an important step, for even if the decision is made and successful surgery is undertaken at this stage it will still be some seven to eight weeks before the patient can return to light work and Io to 12 weeks before he can return to heavy manual labour, if not longer. It is important not to let conservative treatment drag on for six, eight or. ten weeks without real improvement and then present the unfortunate and often demoralized patient with the prospect of another two or even three months following operation before he can resume work. Therefore, if there has been little response to bed rest at the expiration of three weeks the position should be explained to the patient and the chances of cure or improvement by surgery on the one hand and the dwindling chances of further conservative treatment on the other defined, and the patient then left to decide what he wants done. If the pain is still very severe, or if the patient has had previous attacks, there is usually little doubt about the answer. However, if the symptoms are not particularly bad, but still capable of preventing work, economic factors may then come into play. If there is now urgent necessity for the patient to work he may prefer to continue with bed rest for a longer time, even at the risk of it being eventually unsuccessful, and likewise the patient who is more apprehensive of surgery than of his pain, or perhaps has a friend who has had an unsuccessful disc operation, will prefer to continue with conservative measures until he finally convinces himself that his symptoms will not clear up without surgery.

\section{Plaster Jacket}

The use of the plaster jacket is reserved (i) for those patients whose symptoms are not severe enough to demand bed rest and who are anxious to remain ambulant, (ii) for continuing immobilization of the spine after a period of recumbency, or (iii) for those unfortunate few who find that their pain is relieved in the erect posture and is made worse by lying down.

The plaster should be applied with the spine in the position it has naturally adopted and there should be no attempt by head traction to straighten the spine. It should fit snugly and is best applied over two layers of stockinette, with protective pads over the iliac crests and the lumbar spinous processes, and should extend in front from the pubes to the upper third of the sternum and at the back from the posterior iliac spines to the angles of the scapulae. It should be trimmed in the groins and axilla to allow a fairly free range of limb movement. The jacket is retained in the first instance for six weeks and then renewed, for by this time it usually. will have worked a little loose and in any event the spine may be taking up a new position as a result of the relief of pain and spasm. The second plaster should continue to be worn for about three weeks after the symptoms have cleared up.

\section{Other Conservative Treatment}

There are some conservative measures which still require mention. One is manipulation of the spine with or without anaesthesia, and although now and again a dramatic cure of sciatic or back pain results (the reason why is not clear), more often the size of the protrusion is sharply increased and a number of patients date the onset of their sciatica from a manipulation for low back pain. A foot drop and even a cauda equina injury is not unknown. The consensus of opinion is that when a patient has unequivocal signs of a prolapsed disc causing either back or sciatic pain it is a procedure best avoided. Epidural injection of saline, with a small proportion of local anaesthetic via the sacral foramen has had its vogue, but it is difficult to see how it can influence the disc protrusion and its usual effect is to relieve the pain for a few hours only and then when the local anaesthetic has worn off the pain returns with undiminished severity.

\section{Surgical Treatment .}

The indications for surgery will be: (i) Failure of an adequate trial of conservative treatment. (ii) Recurrent attacks of pain. Operation is usually advised at the start of a second attack if the first episode has been severe, but the decision must be left to the patient, who may wish to give conservative treatment a further trial. (iii) Cauda equina compression or severe foot drop. With regard to sciatica the indications under (i) and (ii) above are quite straightforward, but when operation is contemplated for a prolapse causing low back pain alone, stricter criteria are necessary.

At one time practically all low back pain was attributed to disc prolapse and a rather uncritical phase of surgery followed, with poor results. Recently more rigid criteria have been adopted, and as a rule few operations are performed for this condition, probably only I per cent. or 2 per cent. of all operations for disc prolapse. Conservative treatment is usually persisted in for much longer than for sciatica, as the pain is not so demoralizing and in only very few cases does it not clear up eventually, Before operation is advised, and this will only be after prolonged conservative therapy, 
it is essential that the symptoms conform to the well-known clinical picture; pain which is exacerbated on coughing and straining; spasm of the spinal muscles with deformity of the spine, usually an obliteration of lumbar lordosis to which may be added some degree of tilt to one or other side. Flexion and extension movements of the spine are restricted whereas lateral flexion and rotation are comparatively free. If operations for back pain are confined to patients presenting these signs the results are as good as those for sciatica.

\section{Pre-Operative Investigations}

Once surgery is decided upon for sciatica or back pain there are some further investigations which should be done.

Straight X-rays of the spine will already have been taken at the time of the original diagnosis, not so much with the idea of demonstrating any change in the intervertebral disc, which often takes a considerable time to appear, but from a point of view of recognizing certain anomalies, i.e. spina bifida, an increase or reduction in the

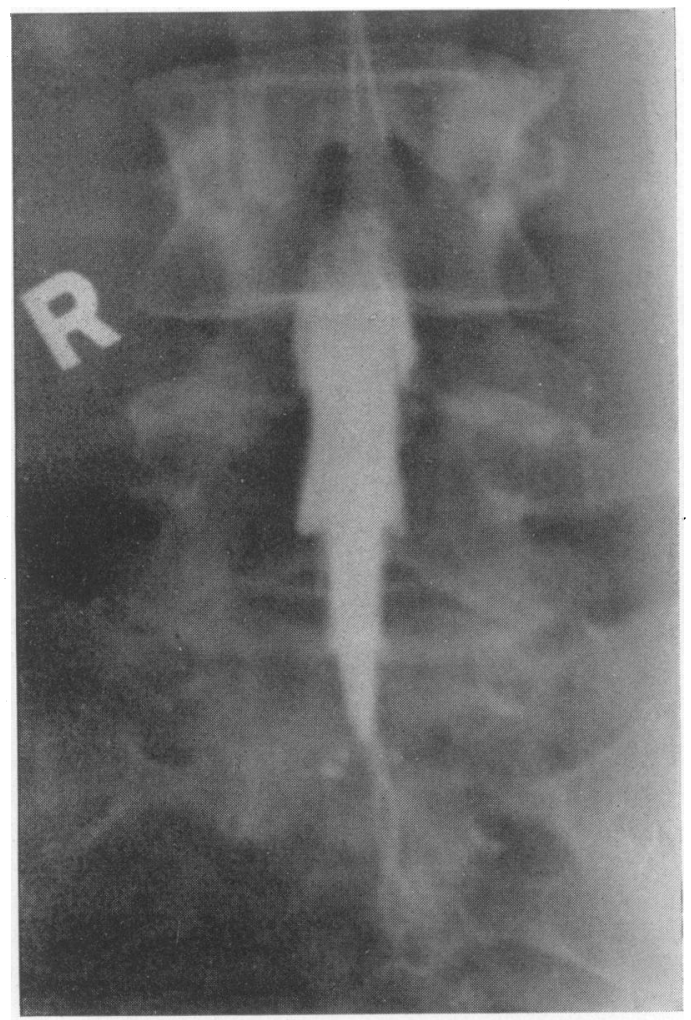

(a)

FIG. 3.-Apparently normal myelogram in a patient who had suffered from left-sided sciatica for one year. Operatiô, was deferred for some months and when eventually performed a very large lumbo-sacral disc prolapse was foured extending from the midline to the intervertebral foramen and involving the first sacral nerve root sheath. number of lumbar vertebrae, or a spondylolisthesi贷 and for excluding more serious conditions such tuberculous infection, primary and secondar tumours of the vertebrae and sacrum, or bone changes resulting from tumours in the spinat: canal.

The next point is whether myelography shoure be undertaken before surgery. This method accurate in about 80 per cent. of cases. The $2 \frac{6}{8}$ per cent. of failures usually take the form of a\& is in fact present, and this more commonly arises with prolapses at the lumbo-sacral level (Fig. 3) This failure is often due to simple anatomicat reasons such as a lateral situation of the protrusiog or the high termination of the dural sac, or a wide anterior extradural space. Clinical diagnosis more reliable than this 80 per cent. success ratê and both the lower disc spaces where 95 per centw of the prolapses occur can be explored quite easily so that myelography is not used as a routine, but reserved (i) for those cases causing a cauda equint syndrome (Fig. 4), (ii) for exclusion or confirmas tion of a suspected tumour, (iii) for localizing at prolapse from one of the higher disc spaces givin rise to anterior crural pain (Fig. 5), and (iv) fớ localizing a prolapse producing only low bacts

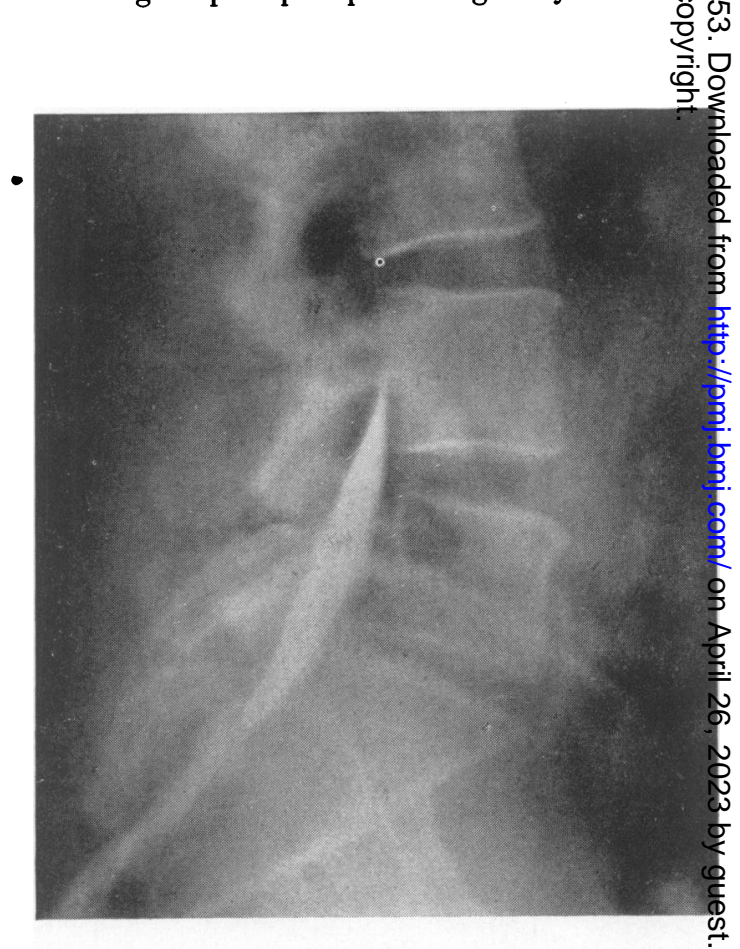

(b) inability to demonstrate a disc prolapse when on 
pain (Fig. 6). As mentioned earlier the protrusions giving rise to back pain may occur in one of several widely separated situations and they are much more difficult to localize clinically than those giving rise to sciatica (for the mere complaint of sciatica immediately localizes the prolapse to the lower two lumbar space discs) so that myelography is of very real value.

One simple investigation before surgery which is well worth carrying out as a routine is that of lumbar puncture and examination of the cerebrospinal fluid, not so much to confirm disc prolapse, but to exclude such occasional causes of sciatica as a cystic arachnoiditis when a rise of cell count will be present, or a spinal tumour when a considerable rise of protein will be observed. As a rough guide, a protein above $100 \mathrm{mg}$. is more suggestive of tumour, such as a neurofibroma or ependymoma, than prolapse, and in the presence of such

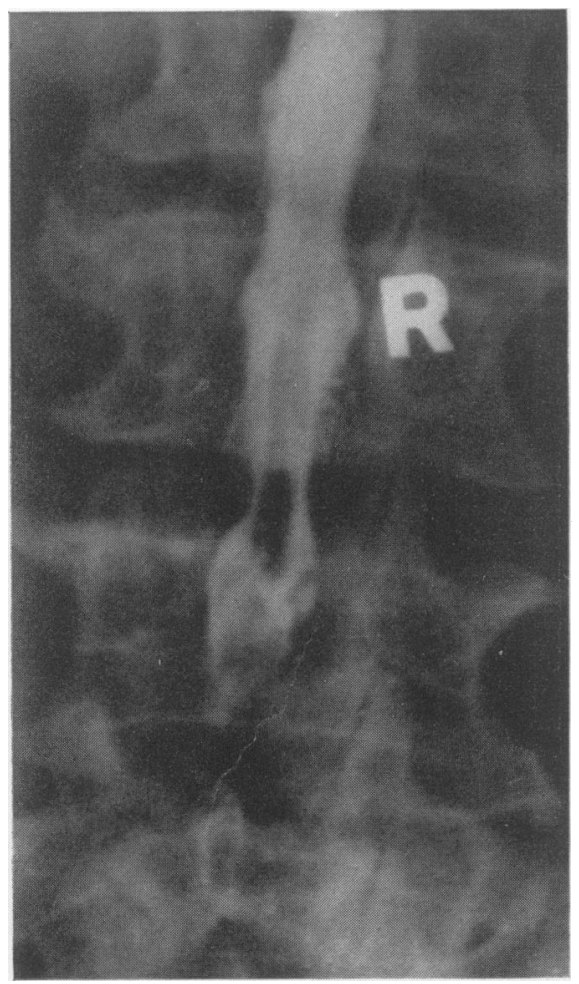

Fig. 4.-Myelogram showing a complete obstruction just above the $L 4 / 5$ disc with the appearance of a conical termination to the theca, indicative of an extra-dural compression, which was due to a massive herniation. The patient had symptoms of a cauda equina lesion which had steadily progressed over two years, simulating a tumour. This is an unusual presentation for a massive prolapse, the majority having an acute onset with a rapid, episodic progression. a figure myelography shoüld be seriously considered.

\section{Operative Technique}

The position of the patient on the operating table is important. Any compression of the abdomen and the intra-abdominal veins will be transmitted to the extradural veins which dilate to a phenomenal size, obscure the prolapse and may give rise to furious bleeding. This can be avoided by operating on the patient either on his unaffected side or, if prone, with supports only under the chest and pelvis so that the abdomen is free. There is some controversy about the exposure of the prolapse, whether it should be done through an interlaminar approach with removal of the ligamentum flavum or through a laminectomy,

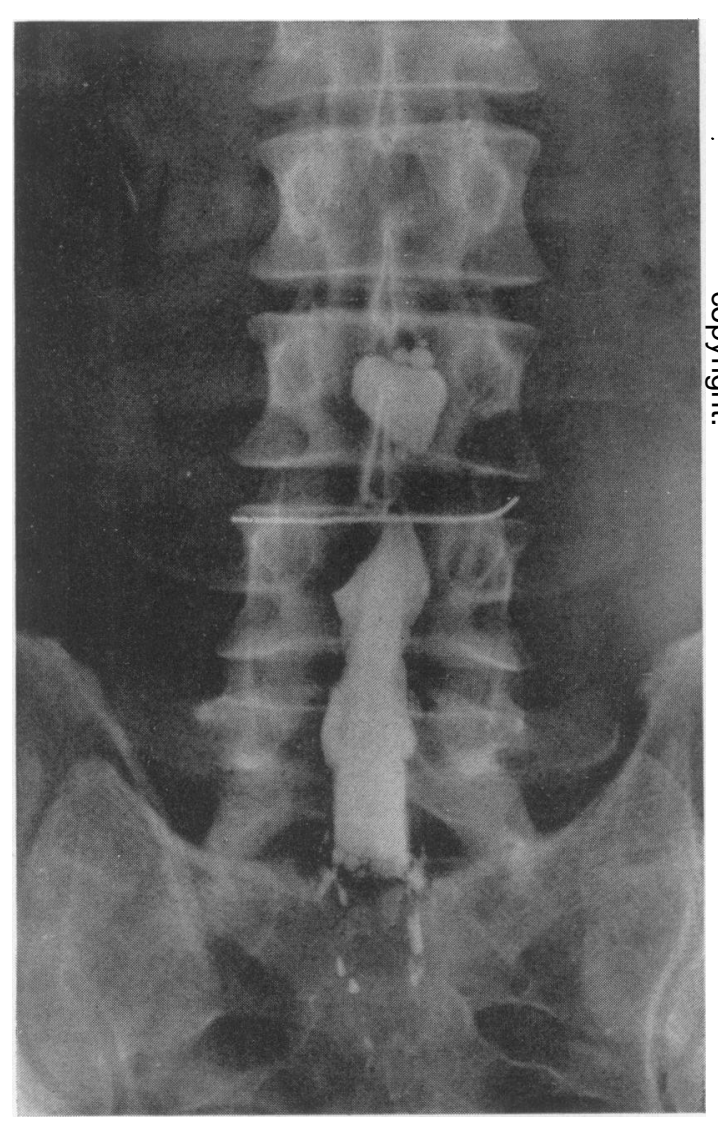

FIG. 5.-Myelogram showing a large filling defect opposite the $\mathrm{L} 3 / 4$ disc, indicating a protrusion which had given rise to anterior crural pain. Myelography is a more reliable investigation for herniations at these higher levels than for the lower two lumbar discs. (The marker wire used pre-operatively to identify the tip of the third lumbar spinous process can also be seen.) 
partial or complete. There should, however, be no rigid rule about this. It will be found that the majority of protrusions of the lumbo sacral joint can be removed through an interlaminar approach but at the $\mathrm{L}_{4} / 5$ level owing to the wider lamina of the fourth lumbar vertebra bone usually needs removal from the adjacent laminal margins, which is best accomplished with an ethmoidal punch forceps. If the opening in either situation is not adequate to expose the prolapse clearly, there should be no hesitation in removing a half lamina or more, for a great deal of harm can be done to the theca and nerve root by too forceful retraction in order to obtain a better view. For a disc prolapse that is known to be centrally placed a hemilamina should be removed at once.

The details of operation are briefly as follows: The fifth lumbar spinous process is identified preoperatively by placing an opaque marker on the skin and taking an X-ray. The incision is then centred over this point. The muscles are separated unilaterally from the spines and laminae, the smooth sloping surface of the sacrum is located as a guide and from this the requisite interlamina space is identified and cleared. The ligamentum

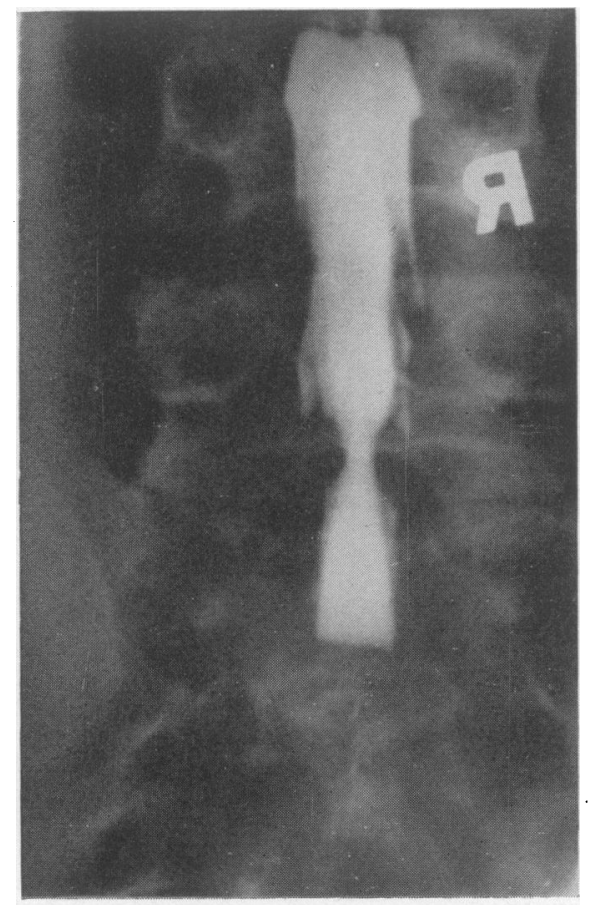

(a) flavum is incised and removed (hinging it and re placing it at the end of the operation as advised by some surgeons has no particular merit) too expose the nerve root and prolapse.

The protrusion may be situated medial or: lateral to, or in front of the root, and is usually $\underset{\vec{s}}{\vec{s}}$ smooth glistening projection covered by the्ठ posterior longitudinal ligament. Sometimes ito will be found to have extruded through the liga $\frac{\bar{s}}{\frac{5}{5}}$. ment and be lying free in the spinal canal as stringy white material, or to have migrated down alonge the nerve root to the intervertebral foramen. Occasionally there may be no obvious protrusion? but only some vascularization of the posterior $\overrightarrow{\vec{\omega}}$ ligament perhaps with the root adherent to it. $\stackrel{\omega}{\rho}$ but on pressure the disc will be found to be soft This variety is often referred to as 'concealed 3 . or 'intermittent' protrusion. The nerve needsin to be accurately identified for it may be stretchedo as a thin band over the top of the prolapse and incised in the belief that it is the capsule, withi resultant damage to some of the fibres and the brisk escape of c.s.f. The prolapse is incised and usually softened portions of mucleus extrude.The opening in the annulus will be seen and en-3 larged and then a thorough curettage of the nucleus carried out with spoon and rongeur so

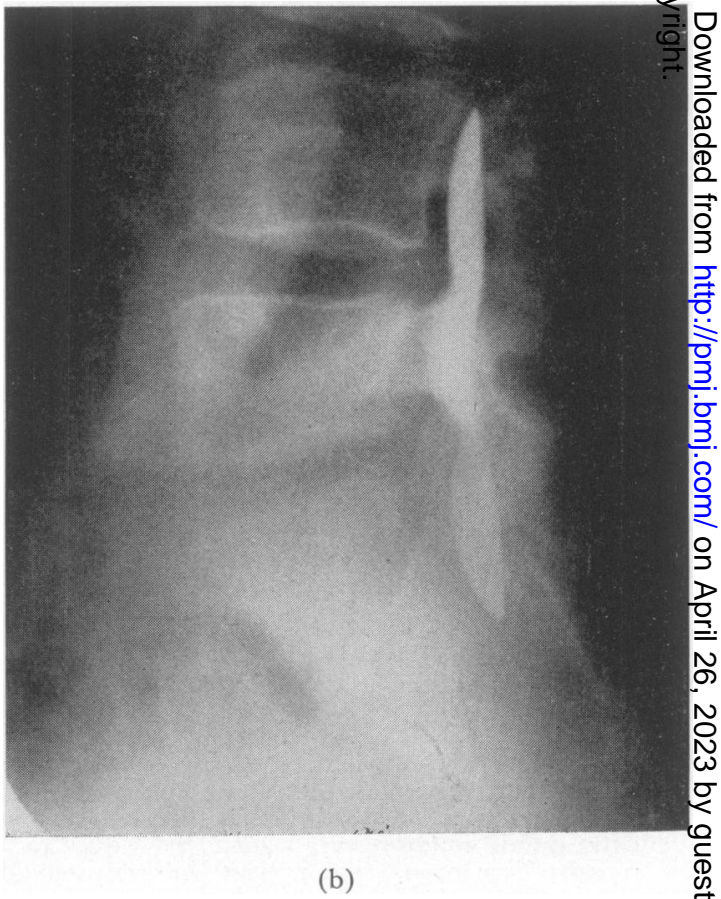

Fig. 6.-Myelogram showing a central prolapse from the lumbo-sacral disc in a patient who suffered from severe and persistent low-back pain without sciatica. This appearance of a narrowed band of contrast material in the midling is thought to be due to the nerve root bundles being displaced to either side off the summit of the prolapse, leaving only a thin channel in the centre for the contrast. 
that there is no loose nuclear tissue left to prolapse at a later date, and the chances of fibrous ankylosis occurring between the vertebrae are increased. Special attention must be paid to portions of the nucleus just anterior to the annulus and lateral to the operative opening in it. If a satisfactory removal cannot be obtained from one side the opposite side should be exposed and the remainder of the nucleus excised. The introduction of instruments into the disc space has to be done with care for there are some reports of damage to the common iliac veins and arteries by an instrument penetrating the anterior portion of the annulus fibrosus. If an unequivocal prolapse is found it is not necessary to explore the disc space above or below, despite the anatomical evidence in cadavers of multiple protrusions, for in practice multiple symptomatic disc protrusions are very uncommon. If, however, there is any doubt whatsoever about the validity of a protrusion, the next space should be explored before the disc is incised. In some cases no prolapse can be found in either space and it is then that the search should be extended far out into the intervertebral foramen, where a small protrusion may be found. It is unnecessary to unroof the foramen by excising the facets forming its posterior wall and thus weakening the spine.

'Negative' explorations do occur, however, and this varies in modern series from 2 to 10 per cent. of cases. Fortunately a proportion even of these patients benefit from exploration, despite the absence of any obvious disc pathology. 'Hypertrophy of the ligamentum flavum' or 'varicosity of the extradural veins' are pathological myths sometimes put forward to explain these successes, but they are merely innocent variations of the normal.

Division of the affected posterior nerve root is sometimes advised but this should never be necessary at a primary exploration. It may be considered in a patient at re-exploration for persistent sciatic pain where the root is found to be extensively adherent, without much evidence of recurrent prolapse.

Another problem is the question of spinal fusion at the time of laminectomy on the eminently reasonable grounds that as one is dealing with a disorganized joint immobilization of it by means of a graft should prevent any back pain after operation. Many varieties of spinal arthrodesis have been devised. One type aims at fusing the lamina and spinous processes with grafts from the ilium or tibia, and this is the popular method in this country. A second variety depends on fusion of the posterior intervertebral articulations, Another one consists of a complete removal of the nucleus and cartilage plates so that the cancellous vertebral surfaces come into close contact, some- times reinforced by the insertion of bone pegs. Yet another variety depends on this same principle but the approach is by an anterior extraperitoneal one to the fronts of the vertebral bodies!

There are, however, many points against $\stackrel{c}{\Rightarrow}$ primary fusion at the time of disc removal: (i) Only few patients have back pain after operation severe enough to incapacitate them, probably only 2 per cent. or 3 per cent. of all operative cases. It is impossible to recognize this small percentage pre-operatively, either on clinical or radiological \& grounds. Often the cases with gross arthritic changes on X-ray have painless backs. (ii) The operation is made more serious and carries a higher risk. (iii) Fusion necessitates the patient staying in bed between 6 to 12 weeks and then wearing a plaster jacket for a further 6 to 3 . 12 weeks, and even then firm union cannot be iु guaranteed. It is therefore the accepted practice $\dot{\omega}$ in this country to remove the prolapse and nucleus pulposus thoroughly and then wait and see what is happens. If severe incapacitating back pain from $\stackrel{\omega}{+}$ intervertebral arthritis still persists after 6 to 12 음 months then a further operation with fusion is justified. Milder back pain is often kept under $\vec{z}$ satisfactory control by using a Goldthwaite corset.

\section{Post-Operative Treatment}

The patient should get up on the seventh tenth day and be ready to leave hospital during the third week.

Back extension exercises can be commenced as soon as the pain from the incision has cleared up, usually about the seventh day. In some clinics $\vec{P}$ spinal exercises are promoted vigorously with the $\frac{0}{3}$ aim of having a full range of spinal movement by the time the patient leaves hospital. This is usually a rather arduous performance for the sufferer and if a long-term view is taken has no 3 . real merit.

No form of support such as a Goldthwaite corset is necessary unless the patient has a good $\delta$ deal of back pain.

The patient should be encouraged to live as 을 normal a life as possible and restriction on his activity should be confined to heavy lifting and any pursuits involving acute flexion of the spine.

\section{Post-Operative Sequelae}

Despite a satisfactory disc removal, some patients are left with residual sciatic pain, which $\widetilde{2}$ is not as a rule as severe as the original pain ando is usually due to fibrosis in the root as a result of $\overparen{D}$ prolonged tension on it by the disc, by too heavy traction at the time of operation or by adhesions $\frac{T}{T}$ which bind the disc to the rent in the annulus and $\underset{\mathbb{D}}{\stackrel{\circ}{*}}$ posterior longitudinal ligament. 
Cramps in the affected leg are quite common and persistent although sciatic pain may have disappeared completely.

A small proportion of patients develop a true recurrent prolapse (prolapse from the same disc) months to years later. This varies in individual statistics, but is probably in the neighbourhood of 4 per cent. to 5 per cent. These recurrent attacks of sciatica, due to a further protrusion, are dealt with in the same way as the primary attacks, except that one will tend to persist with conservative treatment for a much longer period before subjecting the patient to a second laminectomy. There is also a 2 per cent. risk of developing a further disc prolapse at another level.

\section{Results of Surgery}

Table I gives the results in disc surgery at the hands of experienced surgeons. The figures are an average gathered from several large series.

The overall results of treatment of sciatica or

\section{TABle I}

TABle Showing the Results of Operation for Prolapsed Lumbar INTERvertebral Disc

Completely relieved of sciatic or back pain. .

Greatly improved, mild sciatic or back pain; at full work

Improved, more severe sciatic or back pain; at full or light work

No improvement, or worse; unable to work

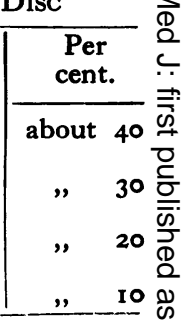

back pain can now be summed up. Out of roo $\vec{\circ}$ patients about 80 will be improved or cured of $\overrightarrow{\vec{\omega}}$ their symptoms by the conservative measures of $\stackrel{\omega}{\sigma}$ bed rest or plaster immobilzation, and 20 will fail 8 to respond. Of these 20 who will eventually come 3 to operation 18 will be made fit to return to work, i and of these eight will be completely relieved of $\dot{\omega}$ symptoms. Two patients will fail to derive any benefit from surgery.

\title{
SUBPHRENIC INFECTION
}

\author{
By A. B. BIRT, F.R.C.S. \\ Norwich
}

The pathology and surgical importance of subphrenic infection cannot be fully understood without a thorough appreciation of the anatomy of the subphrenic space.

\section{Anatomy}

The subphrenic space is defined as being that portion of the abdomen which lies between the diaphragm above and the transverse colon and mesocolon below.

The space is divided into two parts by the liver; the supra-hepatic and infra-hepatic portions.

The supra-hepatic portion is divided into right and left sides by the falciform ligament of the liver. The right side is itself divided into an anterior and posterior space by the right lateral ligament of the liver. The left lateral ligament of the liver runs very close to the posterior margin of the left lobe of the liver so that it does not divide the left suprahepatic area but forms the posterior part of the boundary between the supra- and infra-hepatic parts on the left side. There is, therefore, only one supra-hepatic space on the left side, In addition there is one small extra-peritoneal space in the supra-hepatic part, namely the bare area of the liver, between the leaves of the lateral and falciform ligaments. The supra-hepatic portion of the subphrenic area therefore contains two intra-peritoneal spaces on the right and one on the left and one small extra-peritoneal space. These spaces are usually referred to as the right superior anterior subphrenic space, the right superior posterior subphrenic space, the left superior subphrenic space and the bare area of the liver.

The infra-hepatic portion is divided into right and left parts by the free edge of the lesser $N$ omentum and the descending part of the duodenum. There is only one space on the right in $\mathcal{O}^{-}$ the infra-hepatic region, but on the left there are $\mathrm{W}$ two spaces, an anterior one and a posterior one, being separated from each other by the stomach 0 and the lesser omentum. All the infra-hepatic $\overparen{D}$ spaces are intra-peritoneal, there is no extra- ? peritoneal space below the liver. The infra- 0 hepatic portion of the subphrenic area therefore $\frac{\vec{d}}{\mathbb{D}}$ contains one space on the right and two spaces on 